\title{
Prevalência de Úlceras Por Pressão em Pacientes Internados em um Centro de Terapia Intensiva de um Hospital de Minas Gerais
}

\section{Prevalence of Pressure Ulcers in Patients Admitted to an Intensive Care Unit of a Hospital in Minas Gerais}

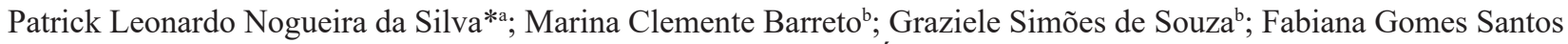 \\ Martins $^{\mathrm{b}}$; Fábio Batista Miranda ${ }^{\mathrm{c}}$; Écila Campos Mota ${ }^{\mathrm{d}}$ \\ anniversidade Federal dos Vales do Jequitinhonha e Mucuri, Programa de Pós-Graduação Stricto Sensu em Saúde, Sociedade e Ambiente. MG, Brasil. \\ ${ }^{\text {b}}$ Faculdades Integradas Pitágoras de Montes Claros, MG, Brasil. \\ ${ }^{c}$ Escola de Saúde Pública de Manaus. AM, Brasil. \\ ¿Universidade Federal de Minas Gerais, Programa de Pós-Graduação Stricto Sensu em Enfermagem. MG, Brasil. \\ *E-mail: patrick_mocesp70@hotmail.com
}

\begin{abstract}
Resumo
As úlceras por pressão são consideradas um importante e considerável problema nos serviços de saúde devido aos custos dessas provenientes, como também pela alta incidência e prevalência, de modo a acarretar consequências irreversíveis em longo prazo, tais como: déficit circulatório, má cicatrização e má perfusão periférica, amputações, entre outras. Sendo assim, este estudo objetivou analisar a prevalência de úlceras por pressão em pacientes internados em um Centro de Terapia Intensiva de um Hospital de Minas Gerais. Trata-se de um estudo descritivo, exploratório, retrospectivo, documental, com abordagem quantitativa, realizado com 151 prontuários de pacientes internados em um Centro de Terapia Intensiva de um hospital durante julho a agosto de 2012. O tratamento dos dados ocorreu por meio de epidemiologia descritiva simples não paramétrica e não probabilística. Observou-se que 51 (33,7\%) destes pacientes desenvolveram úlceras por pressão, com prevalência do sexo feminino, idade superior a 60 anos e tempo de permanência hospitalar inferior a 10 dias. O mês de agosto apresentou maior quantitativo de internações e de pacientes com úlceras $(48,3 \%)$, sendo as mulheres as que desenvolveram mais úlceras do que os homens. As condições ou doenças de base mais prevalentes foram: pós-operatório neurológico $(25,8 \%)$, pós-operatório ortopédico $(11,9 \%)$ e a pneumonia $(7,2 \%)$. Portanto, houve um aumento considerável destas úlceras em pacientes de um Centro de Terapia Intensiva, com prevalência em idosos com tempo de internação menor que 10 dias, quando comparados aos demais pacientes internados, de modo a contribuir para o aumento dos fatores de risco e se tornando indispensável a execução rigorosa do plano de cuidados para cada paciente dentro do setor.
\end{abstract}

Palavras-chave: Lesão por Pressão. Unidades de Terapia Intensiva. Cuidados Críticos.

\begin{abstract}
Pressure ulcers are considered an important and considerable problem in health services due to their costs, as well as their high incidence and prevalence, leading to irreversible long-term consequences such as circulatory deficit, poor healing and poor peripheral perfusion, amputations, among others. Thus, this study aimed to analyze the prevalence of pressure ulcers in patients admitted to an intensive care center of a Hospital in Minas Gerais. This is a descriptive, exploratory, retrospective, documentary study with a quantitative approach, conducted with 151 medical records of patients admitted to an intensive care center of a hospital from July to August 2012. Data were treated through simple nonparametric and non-probabilistic descriptive epidemiology. It was observed that 51 (33.7\%) of these patients developed pressure ulcers, with female prevalence, age over 60 years and hospital stay less than 10 days. The month of August presented the highest number of hospitalizations and patients with ulcers (48.3\%), being women the ones who developed more ulcers than men. The most prevalent underlying conditions or diseases were: neurological postoperative (25.8\%), orthopedic postoperative (11.9\%) and pneumonia (7.2\%). Therefore, there was a considerable increase of these ulcers in patients of an intensive care center, with prevalence in the elderly with hospitalization time fewer than 10 days, when compared to other hospitalized patients, in way that it contributes to the risk factors and, thus, becoming indispensable for the rigorous execution of the care plan for each patient in the sector.
\end{abstract}

Keywords: Pressure Ulcer. Intensive Care Units. Critical Care.

\section{Introdução}

Os avanços da ciência e tecnologia nos tratamentos dos pacientes hospitalizados implicam em uma melhor qualidade assistencial e contribui para o progresso dos mesmos. Esta melhoria não depende somente dos avanços tecnológicos e científicos, mas também do conhecimento dos profissionais envolvidos (COSTA, 2003). Considera-se, segundo Araújo et al. (2010), a pele como sendo o maior órgão do organismo humano, tendo, em média, extensão de $2 \mathrm{~m}^{2}$ no adulto, peso aproximado de $2,7 \mathrm{~kg}$, espessura que varia de $1-4 \mathrm{~mm}$, e que recebe 1/3 do volume de sangue. Santos e Empis (2011) complementam que a pele tem tanta importância como qualquer outro órgão e que nem todos os profissionais da saúde reconhecem a sua importância para a saúde geral e, por isso, muitas das suas alterações ocorrem em função de várias agressões ao longo do tempo.

De acordo com Gomes et al. (2011), as úlceras por pressão (UP) são, geralmente, definidas como áreas de morte celular que ocorrem localizadas sobre proeminências ósseas expostas à pressão por um período de tempo suficiente para causar isquemia. Matos, Duarte e Minetto (2010) enfatizam que as UP são consideradas um importante e considerável problema nos serviços de saúde, em função dos custos delas provenientes, 
como também pela alta incidência e prevalência. Segundo Blanes et al. (2004), as UP ocorrem decorrentes de fatores intrínsecos e extrínsecos. Bavaresco, Medeiros e Lucena (2011) completam que entre os fatores de risco mais relevantes para os pacientes desenvolverem as UP, incluem: déficit de mobilidade e/ou sensibilidade, fricção e cisalhamento, edema, umidade, idade, doenças sistêmicas, medicação, deficiência nutricional, comprometimento neurológico e distúrbios metabólicos.

Giglio, Martins e Dyniewicz (2007) ratificam que a mobilidade reduzida atinge a capacidade do paciente aliviar a pressão, se o mesmo estiver restrito ao leito, de modo a facilitar a fricção e o cisalhamento. Costa (2003), conforme seus conhecimentos sobre UP, relata que a força de fricção e o cisalhamento são comuns em pacientes internados no Centro de Tratamento Intensivo (CTI) em função de os mesmos estarem acamados, instáveis e com alterações dos níveis de consciência, os quais não conseguem movimentar sozinhos, arrastando seu corpo sobre a superfície de apoio ou arrastados pela equipe. Rolim et al. (2013), em seu estudo, afirma que na Unidade de Terapia Intensiva (UTI), além das limitações da atividade e da mobilidade impostas ao paciente pela sua condição clínica, somam-se outros fatores de riscos ligados a tratamentos que exigem um grau de complexidade maior em função da necessidade de haver controles mais rigorosos do seu estado de saúde, como o uso de sedativos, alterações do nível de consciência, uso de drogas vasoativas e instabilidade hemodinâmica que os tornam propensos a UP.

A umidade mantida na pele devido à incontinência urinária e fecal promove um ambiente favorável à proliferação bacteriana, além de fissuras na pele que resultam em feridas, provenientes da pressão que está sendo exercida pela proeminência óssea, favorecendo, assim, o desenvolvimento de UP e progressão das mesmas (WADA; TEIXEIRA NETO; FERREIRA, 2010). De acordo com Costa (2003), o estado nutricional do paciente reflete seu padrão alimentar habitual. Perroni et al. (2011) diz que pacientes que se encontram desnutridos possuem alterações metabólicas, prejudicando assim a cicatrização e fragilização dos tecidos. Segundo Matos, Duarte e Minetto (2010), em função da alta incidência, prevalência, e a diversidade de medidas preventivas, bem como o aumento da morbimortalidade e gastos referente às UP, estas estão entre os grandes problemas encontrados na área da enfermagem.

As UP são frequentes em pacientes graves e por serem considerados efeitos adversos, influencia diretamente na qualidade de vida (QV) do paciente e no cuidado a ele prestado (MATOS; DUARTE; MINETTO, 2010). Cremasco et al. (2009) relatam que nos CTI é elevada a necessidade de cuidados especializados e de alta tecnologia e que diante disso cabe aos profissionais de saúde estabilizarem não só a situação crítica do paciente, como também a manutenção da integridade da pele do mesmo. Nesse contexto, a avaliação do risco para UP pelos profissionais e a implantação de medidas preventivas a esta lesão se torna fundamental.

Justifica-se a importância deste estudo o fato de que as UP, direta ou indiretamente, influenciam na sobrevida e QV de seu portador em longo prazo, de modo a se tornar um risco o seu desenvolvimento. Com isso, o monitoramento constante deste paciente no que diz respeito aos seus fatores de risco para aquisição ou desenvolvimento destes UP deve ser realizado por profissionais de saúde capacitados, a fim de reduzir as comorbidades do paciente, de modo a maximizar o tratamento das UP e conferindo melhoria na QV do paciente.

Sendo assim, este estudo objetivou analisar a prevalência de UP em pacientes internados em um CTI de um hospital de Minas Gerais.

\section{Material e Métodos}

Trata-se de um estudo descritivo, exploratório, retrospectivo, documental, com abordagem quantitativa, realizado no CTI adulto do Hospital Aroldo Tourinho (HAT), localizado no município de Montes Claros, Minas Gerais. O CTI do HAT possui 10 leitos e recebe pacientes com etiologias e condições clínicas e operatórias variadas, com exceção de pacientes pós-operatório cardíaco em decorrência de a instituição apresentar um CTI cardiológico.

Foi enviada uma carta de apresentação e um Termo de Consentimento Institucional (TCI) à Direção Clínica do HAT para autorização do estudo. A instituição foi devidamente orientada quanto às diretrizes da pesquisa e a mesma assinou o TCI de modo a autorizar a realização da pesquisa. A coleta de dados foi realizada no $1^{\circ}$ semestre de 2013, durante os meses de abril e maio, pelo pesquisador responsável.

Foram adotados os seguintes critérios de inclusão para participação na pesquisa: pacientes com, no mínimo, 18 anos de idade ou mais; ter internação compreendida entre o período de $1^{\circ}$ de julho a 31 de dezembro de 2012; ter tempo de permanência de internação no setor de, no mínimo, 24 horas; ter disponível no prontuário todas as informações/dados estipulados para a coleta.

Com relação ao período da internação dos pacientes, foram pesquisados 191 prontuários de pacientes internados no CTI, porém, destes, apenas 151 compuseram a amostra do estudo. Sendo assim, 40 prontuários foram excluídos da amostra por não se enquadrarem aos critérios de inclusão, sendo esses: um paciente ter idade inferior a 18 anos; e 39 pacientes apresentarem tempo de permanência inferior a 24 horas.

A pesquisa foi realizada por meio de dados secundários presentes nos arquivos do Gerenciamento de Risco do CTI, no qual foi utilizada a ficha de admissão preenchida pelo enfermeiro responsável no momento da admissão do paciente no setor. Os dados referentes à entrada, à saída e ao diagnóstico dos pacientes foram coletados nos Censo diário do CTI adulto. 
Os dados foram armazenados no banco de dados Statistical Package for the Social Sciences (SPSS®), versão 20.0. Os mesmos foram tabulados e apresentados em tabelas através de freqüências absolutas, percentuais e medidas de tendência central (MTC), sendo essas a média aritmética ponderada (MAP) e o desvio padrão (DP), na qual se utilizou o programa Microsoft Excel®, versão 2010, para a construção das mesmas. O tratamento dos dados ocorreu por meio de epidemiologia descritiva simples não paramétrica e não probabilística. As variáveis utilizadas para a realização do estudo foram: sexo, idade, tempo de permanência, número de pacientes internados no mês, número de UP e doença de base.

O estudo obedeceu aos preceitos éticos estabelecidos pela Resolução $n^{\circ}$ 466/2012, do Conselho Nacional de Saúde (CNS), na qual regulamenta a realização de pesquisa envolvendo seres humanos (BRASIL, 2016). O projeto de pesquisa foi apreciado e aprovado pelo Comitê de Ética em Pesquisa das Faculdades Integradas Pitágoras de Montes Claros (CEP FIPMoc), sob parecer consubstanciado $\mathrm{n}^{\circ}$ 255.881/2013, Certificado de Apresentação para Apreciação Ética (CAAE) no 15002013.1.0000.5109.

\section{Resultados e Discussão}

Observou-se prevalência de internação do sexo masculino (51,6\%), faixa etária superior a 60 anos $(66,4 \%)$, com média de idade de 62,33 anos, e tempo de permanência durante a internação de até 10 dias (63,5\%) (Quadro 1). Esta prevalência é baseada no quantitativo total da amostra pesquisada $(n=151)$. Nota-se que os pacientes internados são majoritariamente idosos, cujo período de longa permanência favorece o aumento dos fatores de risco para o desenvolvimento de lesões cutâneas (LINI; PORTELLA; DORING, 2016).

Quadro 1 - Aspectos socioeconômicos e permanência de internação da amostra. Montes Claros, Minas Gerais, 2013. $(\mathrm{n}=151)$

\begin{tabular}{|c|c|c|c|}
\hline Variáveis & $\mathbf{n}$ & $\mathbf{\%}$ & MAP+DP \\
\hline Sexo & & & \\
\hline Masculino & 78 & 51,6 & $75,50 \pm, 500$ \\
\hline Feminino & 73 & 48,4 & \\
\hline $18-31$ & 08 & 5,2 & $62,33 \pm 16,61$ \\
\hline $32-45$ & 10 & 6,6 & \\
\hline $46-59$ & 33 & 21,8 & \\
\hline$>60$ & 100 & 66,4 & \\
\hline $\begin{array}{c}\text { Tempo etária (anos) } \\
\text { permanência }\end{array}$ & & & \\
\hline$\leq 10$ dias & 96 & 63,5 & $5,69 \pm 52,765$ \\
\hline $11-20$ dias & 29 & 19,2 & $15,65 \pm 27,702$ \\
\hline $21-30$ dias & 13 & 8,6 & $25,23 \pm 31,336$ \\
\hline $31-40$ dias & 06 & 3,9 & $35,50 \pm 23,774$ \\
\hline $41-50$ dias & 02 & 1,3 & $45,50 \pm 4,500$ \\
\hline$>50$ dias & 04 & 3,5 & $75,25 \pm 10,591$ \\
\hline
\end{tabular}

Fonte: Dados da pesquisa.

Em relação ao sexo, apesar de haver predominância no sexo feminino, esse fator representa uma característica singular da amostra analisada. Rockenbach et al. (2012) observaram prevalência de UP em 27 (49,1\%) dos 55 homens e em 24 $(57,1 \%)$ das 42 mulheres, mas sem diferença significativa entre os grupos. Já Gomes et al. (2011) em seu estudo encontraram $40 \%$ das UP em homens e 30\% em mulheres. É importante destacar que ambos os autores deixaram claro que o sexo não é um fator determinante para o desenvolvimento de UP, e que a prevalência encontrada em seus estudos foi devido ao número de homens e mulheres internados no período da pesquisa. Não foram encontrados outros dados na literatura que relacionem a presença de úlcera com o sexo.

No que se refere à idade, Blanes (2004) relata que a idade avançada provoca várias alterações nutricionais, metabólicas, vasculares e imunológicas, que quando associadas às doenças crônicas (DC), tornam o indivíduo mais propício ao trauma e à infecção. A autora ainda afirma existir maior incidência de feridas crônicas em pacientes na faixa etária acima de 60 anos. Segundo Creutzberg et al. (2011), a idade elevada vem acompanhada de várias limitações, como visuais, auditivas, motoras e intelectuais, que contribuem para o surgimento de doenças crônico-degenerativas (DCD) e as intensificam, ocasionando a diminuição da condição de saúde desses pacientes. Os idosos apresentam maiores condições de risco para o agravamento do quadro inicial e, dessa forma, aumentam a chance para desenvolvimento de UP.

No presente estudo realizado, 66,4\% dos pacientes admitidos no período proposto são idosos de modo a possuir a idade superior a 60 anos, fator este que predispõe e aumenta o risco para o desenvolvimento de UP Creutzberg et al. (2011), justifica o resultado da pesquisa ao afirmar que o envelhecimento ocorre de forma progressiva e irreversível, causando assim desgastes na maior parte dos sistemas funcionais do corpo.

Em relação ao tempo de permanência, Goulart et al. (2008) ratificam que pacientes que permanecem por um período longo confinados ao leito, e que apresentam dificuldades sensoriais ou motoras, imobilidade ou outro problema relacionado a essa condição, apresentam um alto risco para desenvolverem UP. A população de idade superior a 60 anos, quando hospitalizada, o tempo de ocupação do leito é maior se comparado ao de outras faixas etárias, contribuindo assim para o aumento do risco de desenvolvimento de UP (ROCKENBACH et al., 2012). As UP apresentam importância significativa, pois $25-33 \%$ dos pacientes já chegam às instituições apresentando algum tipo de UP e quase $35 \%$ dos pacientes certamente as desenvolverão em algum tempo de sua permanência (COSTA et al., 2005). Relacionado a essa afirmação, Fernandes (2005) infere que as UP constituem uma das complicações que poderão surgir em pacientes que se encontram internados no CTI.

Entre o período abordado nesta pesquisa, o mês de maior prevalência para o acometimento destas afecções foi em agosto (48,3\%), seguido dos meses de julho (33,3\%) e outubro (31,5\%) (Quadro 2). 
Quadro 2 - Prevalência de UP segundo quantidade de internações por mês. $(n=151)$

\begin{tabular}{|c|c|c|c|}
\hline \multirow{2}{*}{ Meses } & \multicolumn{3}{|c|}{ Variáveis } \\
\cline { 2 - 4 } & $\begin{array}{c}\mathbf{N}^{\mathbf{0}} \text { de } \\
\text { internações } \\
\text { (n) }\end{array}$ & $\mathbf{N}^{\mathbf{0}}$ de UP & $\begin{array}{c}\text { Prevalência } \\
\text { de UP (\%) }\end{array}$ \\
\hline Julho & 21 & 07 & 33,3 \\
\hline Agosto & 31 & 15 & 48,3 \\
\hline Setembro & 20 & 05 & 25,0 \\
\hline Outubro & 19 & 06 & 31,5 \\
\hline Novembro & 30 & 09 & 30,0 \\
\hline Dezembro & 30 & 09 & 30,0 \\
\hline Total & $\mathbf{1 5 1}$ & $\mathbf{5 1}$ & $\mathbf{3 3 , 7}$ \\
\hline
\end{tabular}

Fonte: Dados da pesquisa.

Rockenbach et al. (2012) dizem que a UTI oferece um risco maior para a ocorrência de UP devido à gravidade dos pacientes que se internam nesse setor e ao maior número de fatores de risco a que se encontra exposto um doente nessa unidade. Os mesmos apresentam nesta unidade riscos de desenvolver UP duplicados, uma vez que há evidências de que essa condição pode surgir depois de algumas horas de imobilidade. Costa et al. (2005) corroboram ao relatarem que as UP podem começar a se desenvolver em 24 horas ou levar até 5 dias para se manifestar. Gomes et al. (2011), em seu estudo sobre prevalência de UP, relatam que a prevalência das mesmas em adultos varia de $3-14 \%$, podendo chegar até $21 \%$ nos CTI. Neste estudo, a prevalência foi de $33,7 \%$, sendo maior do que a encontrada na literatura.

Do total da amostra estudada $(\mathrm{n}=151), 51$ pacientes $(33,7 \%)$ desenvolveram UP. Entre os pacientes com diagnóstico de UP, o sexo feminino, de modo geral, mostra maior prevalência de acometimento para esta afecção $(51,0 \%)$. Em escala mensal, no mês de agosto, o qual detém o maior quantitativo de pacientes com UP, o sexo feminino também apresentou maior prevalência de casos $(15,6 \%)$, quando comparado ao sexo masculino, conforme mostra claramente no Quadro 3, o qual relaciona a prevalência de UP segundo sexo e meses de ocorrência. Já nos meses de novembro e dezembro houve a inversão desses acometimentos, de modo a prevalecer o sexo masculino em ambos ( $9,8 \%$ e $11,8 \%$, respectivamente).

Quadro 3 - Prevalência de UP segundo sexo e meses de ocorrência. Montes Claros, Minas Gerais, 2013. $(n=51)$

\begin{tabular}{|c|c|c|c|c|c|c|}
\hline \multirow{2}{*}{ Meses } & \multicolumn{2}{|c|}{ Masculino } & \multicolumn{2}{c|}{ Feminino } & \multicolumn{2}{|c|}{ Total } \\
\cline { 2 - 7 } & $\mathbf{n}$ & $\mathbf{\%}$ & $\mathbf{n}$ & $\mathbf{\%}$ & $\mathbf{N}$ & $\mathbf{\%}$ \\
\hline Julho & 03 & 5,8 & 04 & 8,0 & 07 & 13,8 \\
\hline Agosto & 07 & 13,8 & 08 & 15,6 & 15 & 29,4 \\
\hline Setembro & 03 & 5,8 & 02 & 4,0 & 05 & 9,8 \\
\hline Outubro & 01 & 2,0 & 05 & 9,8 & 06 & 11,8 \\
\hline Novembro & 05 & 9,8 & 04 & 7.8 & 09 & 17,6 \\
\hline Dezembro & 06 & 11,8 & 03 & 5,8 & 09 & 17,6 \\
\hline Total & $\mathbf{2 5}$ & $\mathbf{4 9 , 0}$ & $\mathbf{2 6}$ & $\mathbf{5 1 , 0}$ & $\mathbf{5 1}$ & $\mathbf{1 0 0 , 0}$ \\
\hline
\end{tabular}

Fonte: Dados da pesquisa.

As doenças de base se constituem em um conjunto de outras patologias pregressas o qual contribui para a piora do atual quadro clínico do paciente. Estas, por sua vez, podem ocasionar condições clínicas prolongadas ao paciente fazendo com que o mesmo reduza a sua mobilidade física durante o tratamento e recuperação, bem como aumentando os riscos para o surgimento de uma UP. Tais condições clínicas podem ser exemplificadas por meio das seguintes situações: pacientes pós-cirúrgicos, pacientes intubados e em sedação contínua, pacientes comatosos, pacientes politraumatizados, pacientes contidos no leito, entre outros.

Durante a admissão médica, houve $36,8 \%$ dos pacientes, que apresentavam outros diagnósticos advindos antes da internação, sendo estes: cardiopatia crônica $(n=10 ; 18,1 \%)$, acidente vascular cerebral $(\mathrm{n}=10 ; 18,1 \%)$, hipertensão arterial sistêmica $(\mathrm{n}=8 ; 14,5 \%)$, insuficiência renal crônica $(\mathrm{n}=6$; $10,9 \%)$, infarto agudo do miocárdio ( $\mathrm{n}=9 ; 16,3 \%)$, neoplasias $(\mathrm{n}=7 ; 12,7 \%)$ e obesidade $(\mathrm{n}=5 ; 9 \%)$. Sendo assim, as doenças de base ou condições clínicas de maior prevalência foram: paciente pós-operatório neurológico $(25,8 \%)$, paciente pósoperatório ortopédico $(11,9 \%)$ e pneumonia $(7,2 \%)$ (Quadro 4).

Quadro 4 - Perfil epidemiológico das doenças de base apresentadas pelos pacientes durante a admissão médica. Montes Claros, Minas Gerais, 2013. $(\mathrm{n}=151)$

\begin{tabular}{|l|c|c|}
\hline Diagnóstico médico (admissão) & $\mathbf{n}$ & $\mathbf{\%}$ \\
\hline Pneumonia & 11 & 7,2 \\
\hline Choque séptico & 08 & 5,2 \\
\hline Hemorragia digestiva alta & 05 & 3,3 \\
\hline Tromboembolia pulmonar & 06 & 3,9 \\
\hline Pós-Operatório (neurologia) & 39 & 25,8 \\
\hline Pós-Operatório (ortopedia) & 18 & 11,9 \\
\hline Cetoacidose diabética & 04 & 2,6 \\
\hline Politrauma & 05 & 3,3 \\
\hline Outros & 55 & 36,8 \\
\hline Total & $\mathbf{1 5 1}$ & $\mathbf{1 0 0 , 0}$ \\
\hline
\end{tabular}

Fonte: Dados da pesquisa.

Em relação aos pacientes da pesquisa, nota-se que, entre as condições clínico-diagnósticas apresentadas, prevaleceram pacientes em pós-operatório neurológico, pacientes em pósoperatório ortopédico e pneumonia (PNM), estando estes entre as condições ou doenças de base mais comuns. É importante relatar que a maioria dos pacientes apresentava mais de um diagnóstico, porém para a pesquisa foi tabulado apenas os diagnósticos principais. Segundo Costa et al. (2005), os pacientes com algum tipo de trauma medular, apresentam lesão das fibras nociceptivas aferentes que são responsáveis pelo estímulo doloroso para mudança de decúbito, tornando este um fator de risco para desenvolvimento de UP.

Diccini, Camaduro e Iida (2009) relatam que pacientes que apresentam algum problema neurológico podem apresentar algumas sequelas, tais como: motoras, paresia ou plegia em um membro ou em um hemicorpo, prejudicando assim sua mobilidade, e como consequência, o paciente fica restrito ao leito. $\mathrm{O}$ mesmo ainda afirma que esses pacientes podem sofrer algumas alterações de sensibilidade dolorosa e tátil (parestesia), prejudicando a percepção de regiões 
que estão sob pressão. Pacientes com diagnóstico de PNM, geralmente, estão em ventilação mecânica (VM) no CTI, e alguns autores, como Giglio, Martins e Dyniewicz (2007) afirmam que esses pacientes por apresentarem mobilidade reduzida e a capacidade de alívio de pressão prejudicada, predispõem ao cisalhamento e a fricção em função da restrição ao leito.

Segundo Diccini, Camaduro e Iida (2009), as UP em pacientes neurológicos podem ocorrer decorrentes de fatores de risco como: imobilidade, incontinência urinária e fecal, alteração do nível de consciência, fraturas e alteração sensitiva e cognitiva. Além disso, alteração do sono e repouso, perfusão tissular alterada e déficit nutricional são características do paciente neurológico consideradas de risco para desenvolver UP. Sendo assim, muitas vezes, o estado geral supracitado leva o paciente a permanecer por longo tempo em cima de um leito, ficando o indivíduo vulnerável a várias complicações e procedimentos.

Com relação aos outros diagnósticos médicos que não foram listados neste estudo, prevaleceram as doenças cardiovasculares, na qual são fatores predisponentes importantes para a aquisição das UP. Sendo assim, nos estudos de Sales, Borges e Donosco (2010), os mesmos corroboram com os achados deste estudo, na qual as doenças do aparelho circulatório são mais prevalentes e informam que agravos como acidente vascular encefálico (AVE), hipertensão arterial ou outras doenças crônicas podem afetar a capacidade perceptiva, a circulação sanguínea, a oxigenação e a mobilidade, favorecendo a formação de UP.

\section{Conclusão}

Portanto, houve aumento considerável dessas úlceras em pacientes de um Centro de Terapia Intensiva, com prevalência em idosos com tempo de internação menor que 10 dias, quando comparados aos demais pacientes internados, de modo a contribuir para o aumento dos fatores de risco e se tornando indispensável para a execução rigorosa do plano de cuidados para cada paciente dentro do setor. É importante que seja feito o diagnóstico precoce das UP, bem como sua prevenção, avaliando os pacientes de uma forma holística e detectando os fatores de riscos presentes nos mesmos. Algumas ações simples como realizar mudança de decúbito com horários definidos, o uso de dispositivos para alívio da pressão em áreas de proeminência óssea, assim como uma observação criteriosa da pele do paciente diariamente contribuem para a prevenção do desenvolvimento das UP.

\section{Referências}

ARAÚJO, C. R. D. et al. A enfermagem e a utilização da escala de braden em úlcera por pressão. Rev. Enferm. UERJ, v. 18, n. 3, p. 359-364, 2010.

BAVARESCO, T.; MEDEIROS, R. H.; LUCENA, A. F. Implantação da escala de Braden em uma unidade de terapia intensiva de um hospital universitário. Rev. Gaúcha Enferm.
Porto Alegre, v. 32, n. 4, p. 703-710, 2011. doi: 10.1590/S198314472011000400010

BLANES, L. et al. Avaliação clínica e epidemiológica das úlceras por pressão em pacientes internados no hospital São Paulo. Rev. Assoc. Méd. Bras. São Paulo, v. 50, n. 2, p. 182-187, 2004. doi: 10.1590/S0104-42302004000200036

BRASIL. Ministério da Saúde. Conselho Nacional de Saúde. Resolução $n^{\circ}$ 466, de 12 de dezembro de 2012. Aprova as diretrizes e normas regulamentadoras para a realização de pesquisa envolvendo seres humanos. Brasília: CNS, 2012.

COSTA, I.G. Incidência de úlcera de pressão e fatores de risco relacionados em pacientes de um centro de terapia intensiva. Ribeirão Preto: Universidade de São Paulo, 2003. doi: 10.11606/D.22.2003.tde-09032004-084518

COSTA, M.P. et al. Epidemiologia e tratamento das úlceras de pressão: experiência de 77 casos. Acta Ortop. Bras. São Paulo, v.13, n.3, p.124-133, 2005. doi: 10.1590/S141378522005000300005

CREMASCO, M.F. et al. Úlcera por pressão: risco e gravidade do paciente e carga de trabalho de enfermagem. Acta Paul. Enferm., v.22, n.esp, p.897-902, 2009. doi: 10.1590/S010321002009000700011

CREUTZBERG, M. et al. Fatores de risco para úlceras de pressão em idosos de unidade de terapia intensiva. Enferm. Foco, v.2, n.2, p.133-136, 2011. doi: 10.21675/2357-707X.2011.v2.n2.112

DICCINI, S.; CAMADURO, C.; IIDA, L. I. S. Incidência de úlcera por pressão em pacientes neurocirúrgicos de hospital universitário. Acta Paul. Enferm., v.22, n.2, p.205-209, 2009. doi: 10.1590/S0103-21002009000200014

FERNANDES, N. C. S. Úlceras de pressão: um estudo com pacientes de unidade de terapia intensiva. Natal: Universidade Federal do Rio Grande do Sul, 2005.

GIGLIO, M.M.; MARTINS, A.P.; DYNIEWICZ, A.M. Análise do grau de dependência e predisposição à úlcera de pressão em pacientes de hospital universitário. Cogitare Enferm., v.12, n.1, p.62-68, 2007. doi: 10.5380/ce.v12i1.8265

GOMES, F.S.L. et al. Avaliação de risco para úlcera por pressão em pacientes críticos. Rev. Esc. Enferm. USP, v.45, n.2, p.313318, 2011. doi: 10.1590/S0080-62342011000200002

GOULART, F.M. et al. Prevenção de úlcera por pressão em pacientes acamados: uma revisão da literatura. Rev. Objetiva, v.4, n.1, p.85-97, 2008.

LINI, E.V.; PORTELLA, M.R.; DORING, M. Fatores associados à institucionalização de idosos: estudo caso-controle. Rev. Bras. Geriatr. Gerontol., v.19, n.6, p.1004-1014, 2016. doi: $10.1590 / 1981-22562016019.160043$

MATOS, L.S.; DUARTE, N.L.V.; MINETTO, R.C. Incidência e prevalência de úlcera por pressão no CTI de um hospital público do DF. Rev. Eletr. Enferm. , v.12, n.4, p.719-726, 2010. doi: 10.5216/ree.v12i4.8481

PERRONI, F. et al. Estado nutricional e capacidade funcional na úlcera por pressão em pacientes hospitalizados. Rev. Nutr., v.24, n.3, p.431-438, 2011. doi: 10.1590/S1415-52732011000300006

ROCKENBACH, C.W.F. et al. Fatores de risco para desenvolvimento de úlceras de pressão em UTI. ConSci Saúde, v.11, n.2, p.249-255, 2012. doi: 10.5585/conssaude.v11n2.3344

ROLIM, J.A. et al. Prevenção e tratamento de úlceras por pressão no cotidiano de enfermeiros intensivistas. RENE, v.14, n.1, p.148-157, 2013.

SALES, M.C.M.; BORGES, E.L.; DONOSCO, M.T.V. Risco e 
prevalência de úlceras por pressão em uma unidade de internação de um hospital universitário de Belo Horizonte. Rev. Min. Enferm., v.14, n.4, p.566-575, 2010. doi: S1415-27622010000400016

SANTOS, I.; EMPIS, C. À flor da pele. Rev. Port. Clín. Geral., v.27, n.1, p.55-56, 2011.

WADA, A.; TEIXEIRA NETO, N.; FERREIRA, M. Úlceras por pressão. Rev. Med. (São Paulo), v.89, n.3/4, p. 170-177, 2010. doi: 10.11606/issn.1679-9836.v89i3/4p170-177 